\title{
Influence of material, surface reconstruction, and strain on diffusion at the Ge(111) surface
}

\author{
Vasily Cherepanov and Bert Voigtländer \\ Institut für Schichten und Grenzfächen, ISG 3, and cni - Center of Nanoelectronic Systems for Information Technology, \\ Forschungszentrum Jülich, 52425 Jülich, Germany
}

(Received 2 September 2003; revised manuscript received 2 December 2003; published 22 March 2004)

\begin{abstract}
The measurement of the two-dimensional island density after submonolayer deposition is used to determine the effect of material, surface reconstruction, and strain on surface diffusion. Specifically prepared strained and relaxed Ge surfaces are used as templates. Scanning tunneling microscopy is used to determine the density of two-dimensional islands. The change of the material (from Si to Ge) increases the diffusion length substantially. The diffusion length is increased by a factor of 10 when the substrate material is changed from Si to Ge. The effect is weaker when the deposited material is changed. The diffusion barrier for Ge and Si adatoms is found to increase with increasing compressive strain of the $\mathrm{Ge}(111)$ substrate. Unexpectedly, the change of the surface reconstruction from $(7 \times 7)$ to $(5 \times 5)$ has negligible influence on the diffusion length.
\end{abstract}

DOI: 10.1103/PhysRevB.69.125331

PACS number(s): 68.55.Ac, 68.35.Fx, 68.37.Ef

\section{INTRODUCTION}

Recently, the heteroepitaxial growth of lattice mismatched semiconductor systems, such as $\mathrm{Ge} / \mathrm{Si}$ and $\mathrm{InAs} / \mathrm{GaAs}$, has attracted substantial interest. Here the surface diffusion during growth is especially important, because it directly affects the film morphology. The surface diffusion will be influenced by almost any property of the surface, by the material (chemical composition), by the surface structure (surface reconstruction), and by the elastic properties of the surface (strain). The challenge is to separate the influence of the various properties of the surface on the diffusion.

The influence of the material ( $\mathrm{Si}$ or $\mathrm{Ge}$ ) on the diffusion length was studied in homoepitaxial growth of $\mathrm{Si}(111)$ and $\mathrm{Ge} / \mathrm{Si}(111)$ and it was found that Ge epitaxy leads to larger diffusion length. ${ }^{1}$ However, it is unclear, if this effect will remains on the substrate of the different material.

The influence of the surface structure on diffusion is known best on the $\mathrm{Si}(001)$ surface. The diffusion parallel to the dimer rows is faster than the diffusion across the dimer rows by a factor of up to $1000 .^{2-5}$ The comparison of the diffusion on two different reconstructions is more difficult, since usually only one specific reconstruction exists on a particular surface like the $(2 \times 1)$ reconstruction on the $\mathrm{Si}(001)$ surface.

The influence of strain on adatom diffusion is important, because in any heteroepitaxial growth strain is involved. There is only a small number of studies that investigated the effect of strain on the surface diffusion barrier. The barrier for the metallic system $\mathrm{Ag}$ on $\mathrm{Ag}(111)$ is found to decrease with increasing compressive strain and increase with tensile strain. ${ }^{6,7}$ Schroeder and Wolf ${ }^{8}$ found the same behavior of diffusion barrier with strain for atoms, which interact by a Lennard-Jones potential. For metals, the strain dependence of the surface diffusion can be understood in an intuitive way. Lattice compression moves the diffusing atoms out so that they experience a less corrugated potential surface. In the case of semiconductors, the strain dependence of the surface diffusion cannot be explained so simply. Recent firstprinciple calculations of the activation energy for $\mathrm{Si}$ adatoms on $(1 \times 1)-\operatorname{Si}(111)$ surface demonstrated ${ }^{9}$ an increase of the barrier when the surface is under compressive strain and a decrease for tensile strain.

In this paper we use the measurement of the twodimensional (2D) island density after submonolayer deposition to study the diffusion on Ge(111) surfaces which are different with respect to surface reconstruction, strain, and material. If possible, we compare the island density on template surfaces which differ only in one of the previously mentioned properties. This is done in order to study selectively the dependence of the diffusion on this property, i.e., surface reconstruction, or strain, or material. It was found that the change of the material (from $\mathrm{Si}$ to $\mathrm{Ge}$ ) increases the diffusion length substantially. The most drastic increase of the diffusion length (factor of 10) is observed when the substrate material is changed from $\mathrm{Si}$ to $\mathrm{Ge}$. The increase of the diffusion length is less pronounced, when the deposited material (the diffusing species) is changed from $\mathrm{Si}$ to Ge. Regarding the influence of strain on diffusion a larger diffusion length was found on a relaxed Ge(111) surface compared to a compressively strained surface. Unexpectedly the influence of the surface reconstruction $((7 \times 7) \leftrightarrow(5 \times 5))$ on the diffusion length is negligible.

\section{EXPERIMENT}

The experiments were performed in an ultrahigh vacuum chamber with a base pressure $p<3 \times 10^{-11}$ mbar. The chamber contains the scanning tunneling microscope (STM) and $\mathrm{Si}$ and $\mathrm{Ge} e$-beam evaporators for deposition of $\mathrm{Si}$ and Ge by molecular-beam epitaxy (MBE). The samples were cut from $\mathrm{Si}(111) \mathrm{Sb}$-doped wafers $\left(\sim 1 \times 10^{19}\right.$ atom $/ \mathrm{cm}^{3}$ doping). Flat clean $\mathrm{Si}(111)-7 \times 7$ surfaces were prepared by an in situ cycles of annealing at $1500 \mathrm{~K}$. The STM images were taken in the constant current mode at sample bias between 2 and $-2 \mathrm{~V}$ and tunneling current of $1 \mathrm{nA}$. A quartz crystal balance and STM images were used to measure the deposited amount of Si and Ge. Si and Ge were evaporated at a rate of 
$1 \mathrm{ML} / \mathrm{min}\left[1\right.$ monolayer $(\mathrm{ML})=7.8 \times 10^{14}$ atoms $\left./ \mathrm{cm}^{2}\right]$. The temperature of the substrate was measured using an infrared optical pyrometer for the high-temperature range ( $T$ $>720 \mathrm{~K})$. An extrapolation of the temperature-power law was used for the low-temperature range $(T<720 \mathrm{~K})$.

\section{2D ISLAND DENSITY AND DIFFUSION BARRIER}

A direct observation of a single atom diffusing jump on a surface using STM is only possible at temperatures close to room temperature, ${ }^{4}$ where the nature of the diffusing species and the diffusion processes can be different from the ones for realistic growth temperatures of several hundred kelvins. Therefore, to study the surface diffusion we need to measure a parameter which relates to the surface diffusion. Such a parameter is the average distance an atom travels before the diffusion is terminated by incorporation into islands or nucleation. This distance we call "effective" diffusion length. In the regime of 2D island growth mode this effective diffusion length is proportional to the average distance between $2 \mathrm{D}$ islands after submonolayer deposition.

The diffusion length depends of course on the activation energy to hop from one binding site to the next $\left(E_{d}\right)$. However, as will be explained now, the diffusion length depends as well on the lateral bonding of atoms in a nucleus of a $2 \mathrm{D}$ island. If several diffusing atoms meet at one point on the surface they form a nucleus. The probability to decay or survive for such a nucleus depends on the lateral bonding inside of the nucleus. If bonding is weak the nucleus will decay easily, if bonding is strong enough the nucleus can grow further to a stable 2D island. More strong lateral bonding between the atoms means less atoms are necessary to form a stable nucleus. The aggregation of a smaller number of atoms happens more frequently and hence nucleation events occur more often in this case. Therefore, for atoms with strong lateral bonding the island density will be higher than for less strong bonded atoms. Often it turns out to be difficult to assign an observed change in the diffusion length unambiguously to one mechanism: modified barrier for diffusion (for instance, due to strain) or modified bonding in the nucleus. If possible, we use additional arguments on the effect of one of the mechanisms to exclude this effect.

To calculate the diffusion barrier quantitatively we used the Venables theory of nucleation. In terms of that model the following parameters define the island density at a given temperature and growth rate $(R)$ : the activation energy of surface diffusion $\left(E_{d}\right)$ and the binding energy $\left(E_{i}\right)$ of the critical cluster of size $i\left[\left(E_{i}\right)\right.$ is a function of the binding energy between a pair of atoms $\left.\left(E_{b}\right)\right]$. Adatoms are assumed as diffusing species. If reevaporation from the substrate can be neglected, as it is the case in MBE growth, the island density $N$ can be written as

$$
N \propto\left(\frac{R}{\nu_{0}}\right)^{p} \exp \left(\frac{E}{k T}\right),
$$

where $E=\left(E_{i}+i E_{d}\right) /(i+2), p=i /(i+2)$, and $\nu_{0}$ is a characteristic surface vibration frequency. ${ }^{10}$ (a)

\begin{tabular}{|c|c|c|c|}
\hline 1BL & Strained Ge & $7 \times 7$ & $5 \times 5$ \\
\hline & $\operatorname{Si}(111)$ & & \\
\hline
\end{tabular}

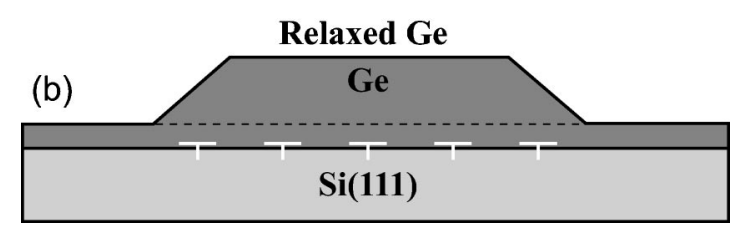

FIG. 1. Schematic view of a strained Ge surface growing pseudomorpically on the Si substrate (a) and of a relaxed Ge surface on top of a relaxed 3D Ge island (b). Such samples are used as a templates of strained and relaxed surfaces.

At constant growth rate the island density is determined by two energies $E_{d}$ and $E_{i}$. We performed the measurements of the island density as a function of temperature to investigate the diffusion on the surface. The slope of the linear fit in the Arrhenius plot of the data gives the value of the activation energy $E$. This activation energy consists of the contributions from two energies $E_{i}+i E_{d}$ or in other words two processes - the diffusion of the atoms and their sticking. Using only island density measurement it is not possible to separate them. However, if we compare the island density on two different templates, the behavior of one of the energies may be intuitively clear and we can qualitatively infer the other one from the experimental data. Such a qualitative result would be the following: The diffusion barrier increases/ decreases on a strained template compared to a relaxed one.

\section{FABRICATION OF STRAINED AND RELAXED SURFACES}

To elucidate the effect of strain on the surface diffusion it is necessary to prepare strained and unstrained surfaces with the same surface reconstruction, because the surface reconstruction itself can influence the diffusion on the surface. Using an epitaxially grown $\mathrm{Ge}$ film on $\mathrm{Si}(111)$ substrate it is possible to create strained and nonstrained surfaces with the same surface reconstruction. Due to the $4 \%$ larger lattice constant of $\mathrm{Ge}$, a solid pseudomorphic film of $\mathrm{Ge}$ on the $\mathrm{Si}(111)-(7 \times 7)$ substrate is under strong compressive stress, see Fig. 1(a). Such a film can be used as a template of the strained surface. Depending on the deposition conditions (see below) a mixture of $(7 \times 7)$-and $(5 \times 5)$-reconstruction domains is found on these strained films [Fig. 1(a)]. The easiest way to obtain an unstrained Ge surface is just to use a Ge bulk sample. However, a bulk Ge sample reveals a different surface reconstruction $c(2 \times 8)$ (Ref. 11) which makes it unacceptable to use it as a template of nonstrained Ge surface due to the unknown effect of this reconstruction on the diffusion. In our experiment we used the known fact that on top of $3 \mathrm{D} \mathrm{Ge}$ islands grown on $\mathrm{Si}(111)$ a large flat area with a $(7 \times 7)$ reconstruction exists. ${ }^{12}$ On the one hand there are several indications that these 3D islands are largely 
relaxed. First, the Ge lattice spacing inside the 3D islands was measured by electron diffraction to be $\sim 70 \%$ relaxed. ${ }^{13}$ Second, we found surface undulations on top of 3D islands indicative of a strain relieving dislocation network at the $\mathrm{Si} / \mathrm{Ge}$ interface. The distance between the dislocation lines indicates a relaxation of $\sim 75 \% .{ }^{14}$ On the other hand the presence of the $(7 \times 7)$ reconstruction indicates that a small residual strain is still present. ${ }^{15}$ In this study the $(7 \times 7)$ surface reconstruction was replaced by the $c(2 \times 8)$ reconstruction for a relaxation larger than $95 \%$. Therefore, such a surface is a source of a nearly unstrained Ge surface. Figure 1 shows a schematic view of the strained (a) and relaxed (b) $\mathrm{Ge}(111)$ surfaces used in the experiments.

A temperature of $770 \mathrm{~K}$ was used to create the compressively strained and the relaxed Ge films. This temperature was chosen on the one hand to create large defect-free areas of the required surfaces, and on the other hand to avoid intermixing.

On the $\mathrm{Si}(001)$ surface it was found that during the initial deposition of $\mathrm{Ge}$ on $\mathrm{Si}(001)$ the incorporation of $\mathrm{Ge}$ is displacive. ${ }^{16}$ In this case the incoming Ge atoms are incorporated randomly into the $\mathrm{Si}$ surface and displace $\mathrm{Si}$ atoms from the surface layer which then diffuse towards the step edges. This displacive incorporation leads to a strong $\mathrm{Si} / \mathrm{Ge}$ intermixing on the $\mathrm{Si}(001)$ surface. As a result of two control experiments we found that $\mathrm{Si} / \mathrm{Ge}$ intermixing is not so strong on the $\mathrm{Si}(111)$ surface.

To elucidate the possible effect of intermixing we compare surfaces which would have a large amount of $\mathrm{Si}$ at the surface in the case of substantial Si/Ge intermixing to a surface which contains only $\mathrm{Ge}$ (also if $\mathrm{Si} / \mathrm{Ge}$ intermixing is substantial). As we will show later, a high amount of Si in the substrate leads to a much higher 2D island density. Since we do not observe this effect when comparing the island densities on our two reference surfaces, we conclude that SiGe intermixing is small for the conditions used here.

In detail, we compare the island densities of $2 \mathrm{D}$ Ge islands on a 2 ML Ge film and on the 6 ML thick Ge wetting layer. If $\mathrm{Si} / \mathrm{Ge}$ intermixing is strong, the $\mathrm{Si}$ content in the 2 ML Ge film will be substantial. This is different for the wetting layer. The wetting layer has a thickness of about $6 \mathrm{ML}$ Ge. Therefore, even in the presence of substantial $\mathrm{Si} / \mathrm{Ge}$ intermixing every consecutively grown layer of Ge should have a lower concentration of Si. Hence, a significantly lower amount of $\mathrm{Si}$ is expected in the top layer of the wetting layer compared to the one layer Ge film. As we will show in a later part of this paper: A large Si content of the substrate should lead to a high density of 2D islands and a large content of Ge in the substrate leads to a lower island density. If we would find a different density of 2D Ge islands on the two surfaces, this would indicate a different Si content. However, experimentally we find almost the same $2 \mathrm{D}$ island density on the $6 \mathrm{ML}$ Ge wetting layer as on the $2 \mathrm{ML}$ Ge film. Figure 2 shows an Arrhenius plot of the island density dependence for epitaxy of $\mathrm{Ge}$ on $(5 \times 5)$ reconstructed $2 \mathrm{ML}$ $\mathrm{Ge}$ and on the Ge wetting layer $[(5 \times 5)$ reconstructed]. As one can see from the figure, the data points for island density on the wetting layer lie only slightly lower than for the deposition on $(5 \times 5)-2$ ML Ge film. Since, for substantial inter-

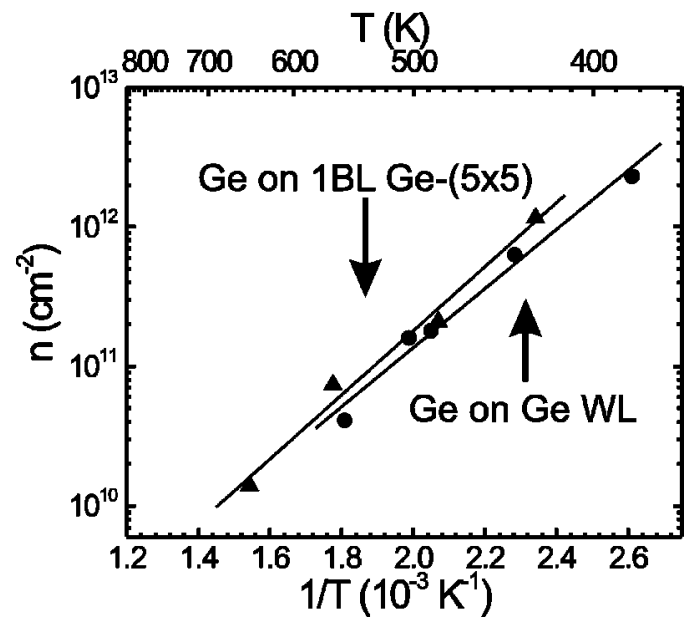

FIG. 2. Island density of $2 \mathrm{D} \mathrm{Ge}$ islands as a function of growth temperature on a $(5 \times 5)$-reconstructed $2 \mathrm{ML} \mathrm{Ge}$ film grown on $\mathrm{Si}(111)$ and on the $6 \mathrm{ML}$ thick Ge wetting layer $[(5 \times 5)$ reconstructed].

mixing, the island density on the 2 ML film would be expected to be $\sim 2.5$ times larger than on the wetting layer (see below), we conclude that no substantial $\mathrm{Si} / \mathrm{Ge}$ intermixing occurs.

Evidence against $\mathrm{Si} / \mathrm{Ge}$ intermixing in the $2 \mathrm{ML}$ Ge film is also found from the areas occupied by $(5 \times 5)$ and $(7$ $\times 7)$ reconstruction domains found for different growth conditions. On a 2 ML thick Ge film both $(5 \times 5)$ and $(7 \times 7)$ surface reconstruction domains are found. ${ }^{12}$ One could assume that the $(7 \times 7)$-reconstructed domain is intermixed with $\mathrm{Si}$ and maintains the $(7 \times 7)$ structure due to a large $\mathrm{Si}$ content. If Si/Ge intermixing does occur in the $2 \mathrm{ML}$ Ge film, one would expect that intermixing would be strongest for low deposition rates. In this case the $(7 \times 7)$ domain (the presumably intermixed phase) should be more prominent, because more time is given for interdiffusion. Conversely, for higher deposition rates intermixing should be kinetically suppressed; due to the high rate less time is available for interdiffusion. This would lead to a larger area of the (5 $\times 5$ )-reconstruction domain. However, in the experiment we find just the opposite, which shows that the $(7 \times 7)$ domain does not form due to a high Si content.

In detail, by choosing certain growth conditions (substrate temperature and deposition rate) it is possible to create a 2 ML Ge film with a surface where one type of reconstruction is dominant. Using variety deposition parameters we found that at lower deposition rate (about $0.03 \mathrm{ML} / \mathrm{min}$ ) the surface of 2 ML film has mostly $(5 \times 5)$ reconstruction. Films deposited at higher rate (about $1 \mathrm{ML} / \mathrm{min}$ ) always reveal a mixture of $(7 \times 7)$ and $(5 \times 5)$ reconstructions. The low deposition rate has higher probability of intermixing due to longer lifetime of adatoms before incorporation to the crystal, and therefore longer time for which adatoms diffuse at the surface. Since for low deposition rate the $(5 \times 5)$ domain forms, which is known to occur for large strains and pure Ge, the experimental results are inconsistent with substantial intermixing. The experiments show that the equilibrium structure forming at low growth rates of $2 \mathrm{ML} \mathrm{Ge}$ is $(5 \times 5)$ recon- 


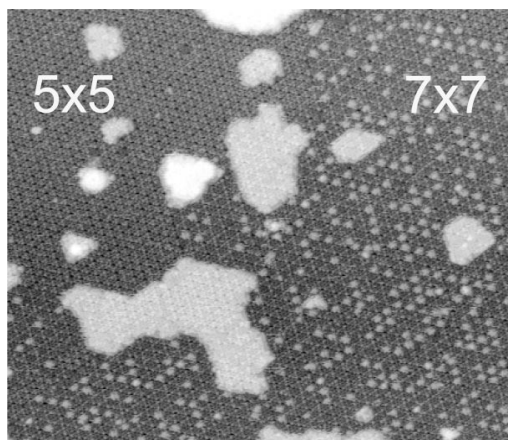

FIG. 3. STM image of $0.4 \mathrm{ML}$ Ge grown at $560 \mathrm{~K}$ on $2 \mathrm{ML} \mathrm{Ge}$ film on $\mathrm{Si}(111)$. Two regions with different reconstructions are imaged.

structed and under kinetic conditions (high growth rate) a metastable $(7 \times 7)$ Ge structure forms.

In summary, we obtained two independent experimental indications that the intermixing of Si into the 2 ML Ge layer is small.

\section{INFLUENCE OF SURFACE RECONSTRUCTION ON DIFFUSION}

A typical substrate temperature of $770 \mathrm{~K}$ and a deposition rate of $0.7 \mathrm{ML} / \mathrm{min}$ have been used to grow a $2 \mathrm{ML}$ Ge film on $\mathrm{Si}(111)$ to obtain a surface, which contains a mixture of relatively large $(7 \times 7)$ and $(5 \times 5)$ surface reconstruction domains. The island density has been measured after submonolayer deposition on both types of reconstructed surfaces. Figure 3 shows a STM image of $0.4 \mathrm{ML}$ Ge grown at $560 \mathrm{~K}$ on a $2 \mathrm{ML} \mathrm{Ge} / \mathrm{Si}(111)$ film. A $(5 \times 5)$-and a $(7 \times 7)$-reconstructed area are clearly visible in the image. There are two types of islands present on the surface. The first type of islands are usual 2D islands, which have a size of several unit cells of the surface reconstruction. The second island type is small islands, so called "magic" clusters. ${ }^{17}$ The size of the magic cluster is smaller than a half unit cell of the surface reconstruction.

From Fig. 3 it is evident, that magic clusters appear only on the $(7 \times 7)$-reconstructed areas and almost no magic clusters are located at $(5 \times 5)$ areas. The magic clusters grow to a certain size and cannot grow larger than this magic size. Every magic cluster is located within a faulted half of the reconstruction unit cell. More detailed experiments show that the density of the magic clusters on the $(7 \times 7)$ part changes significantly with temperature. At low-temperature epitaxy $(430 \mathrm{~K})$ the surface has an extremely high density of magic clusters, more than $50 \%$ of faulted half unit cells are occupied. At higher temperatures $(650 \mathrm{~K})$ the density of magic clusters is lower and the deposited material nucleates as larger 2D islands. Also for the $(5 \times 5)$ surface a qualitatively similar trend is observed. The density of magic clusters on the $(5 \times 5)$ is negligible in the temperature above $400 \mathrm{~K}$. Whereas a significant number of magic clusters can be observed on the $(5 \times 5)$-reconstructed Ge surface at deposition temperature below $300 \mathrm{~K}^{18}$ The magic clusters on the (5 $\times 5$ )-reconstructed surface are also mostly located on the

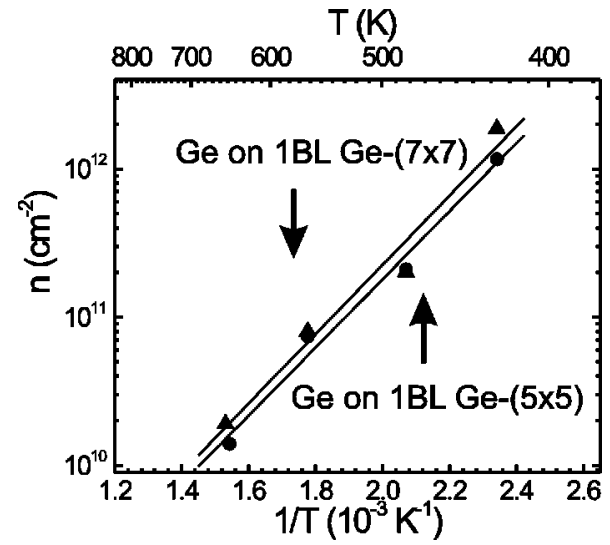

FIG. 4. Island density of $2 \mathrm{D}$ Ge islands as a function of growth temperature on the $(5 \times 5)$ - and the $(7 \times 7)$-reconstructed $2 \mathrm{ML} \mathrm{Ge}$ film grown on $\mathrm{Si}(111)$.

faulted half of the $5 \times 5$ unit cell. These results show that the magic clusters are stable at low temperatures $[300 \mathrm{~K}$ on the $(5 \times 5)$ surfaces and $430 \mathrm{~K}$ on the $(7 \times 7)$ surfaces, respectively]. At high temperatures the magic clusters are not stable and larger 2D islands form. In the following we explain why the magic clusters are stable up to much higher temperatures on the $(7 \times 7)$ surface compared to the $(5 \times 5)$ surface (Fig. $3)$. The area of the $(7 \times 7)$ surface unit cell is almost twice as large as the area of the $(5 \times 5)$ unit cell. This means that also the number of atoms in the magic cluster is much larger on the $(7 \times 7)$ surface than on the $(5 \times 5)$ surface. The larger a cluster is, the larger will be the binding energy and the more stable this cluster will be. The absence of the magic clusters on the $(5 \times 5)$ surfaces for temperatures larger than $400 \mathrm{~K}$ is explained by the instability of this small clusters at temperatures exceeding $400 \mathrm{~K}$. The larger magic clusters on the (7 $\times 7)$ surface are (meta)stable up to higher temperatures.

In spite of the fact that the density of the magic clusters is very different on the two reconstructions, we find, surprisingly, that the density of the larger $2 \mathrm{D}$ islands is very similar on the $(7 \times 7)$-and on the $(5 \times 5)$-reconstructed surfaces. On these surfaces it is possible to find relatively large areas without surface defects, such as reconstruction domain boundaries or pits. The islands on large domains which are located far from a domain boundary were counted to measure the island density. Figure 4 shows the temperature dependence of the $2 \mathrm{D}$ island density for $\mathrm{Ge} 2 \mathrm{D}$ islands grown on the $(5 \times 5)$ - and $(7 \times 7)$-reconstructed $2 \mathrm{ML} \mathrm{Ge}$ film on $\mathrm{Si}(111)$. In the temperature range between $400 \mathrm{~K}$ and $700 \mathrm{~K}$ the difference in the island density on the $(5 \times 5)$ - and $(7 \times 7)$-reconstructed film is almost negligible. This means nucleation of 2D islands happens with the same frequency on $(5 \times 5)$ and $(7 \times 7)$ surfaces, in spite of the large difference in surface morphology [presence of magic clusters on (7 $\times 7)$-and absence of the magic clusters on the $(5 \times 5)$-reconstructed areas].

There are two potential effects influencing the diffusion length: First the presence of small clusters on $(7 \times 7)$-reconstructed surface should reduce the diffusion length, and second the presence of trenches between the half unit cells on the DAS (dimer adatom stacking fault) recon- 
structed surface should increase the diffusion length. When we look at the morphology of the DAS reconstructed (7 $\times 7)$ and $(5 \times 5)$ surfaces, they consist of triangular subunits terminated by adatoms, trenches between the subunits formed by dimers, and the corner holes. It is known that the diffusion inside the triangular subunits of the DAS reconstruction is easy, while the diffusion from triangular subunit to triangular subunit (across the trenches formed by the dimers) has a much higher barrier. ${ }^{19}$ Due to the smaller unit cell of the $(5 \times 5)$ reconstruction the density of trenches is larger on this surface and a smaller diffusion length is expected due to diffusion over more trenches per unit length on this surface than on the $(7 \times 7)$ surface. On the other hand the presence of the small clusters on the surface also could influence the diffusion over this surface. The presence of the magic clusters on $(7 \times 7)$-reconstructed surface should effectively reduce the number of available sits for diffusing atoms, what should decrease the effective diffusion length on $(7 \times 7)$ relative to that on the $(5 \times 5)$-reconstructed surface.

One might think that these two effects may compensate. However, the temperature dependence of both effects is different. The density of the small clusters is found experimentally to be temperature dependent (not shown), whereas the density of trenches does not depend on temperature. Therefore, a mutual cancellation of both effects is not possible over an extended temperature range and both effects have to be small effects individually. In summary, the very similar density of 2D islands on both the $(7 \times 7)$ - and $(5 \times 5)$-reconstructed surfaces indicates that the effective diffusion is, unexpectedly, independent of the reconstruction.

\section{INFLUENCE OF STRAIN ON DIFFUSION}

A deposition rate of $1 \mathrm{ML} / \mathrm{min}$, a surface temperature of $770 \mathrm{~K}$, and a coverage of $15 \mathrm{ML}$ have been used to obtain 3D Ge islands with a flat top consisting of a $(7 \times 7)$-reconstructed surface. These islands are largely strain relaxed. Figure 5(a) shows a typical STM image of a 3D Ge island with subsequent deposition of $0.3 \mathrm{ML}$ Ge. There are some defects, such as domain boundaries and stacking faults, but it is possible to find 3D islands, which have large areas of nondefected $(7 \times 7)$-reconstructed surface. In Fig. 5(b) a close view of the same island is presented. Series of experiments with deposition of submonolayer $\mathrm{Si}$ and $\mathrm{Ge}$ on such relaxed $\mathrm{Ge}$ surfaces have been performed.

Figures 6(a) and 6(b) show two STM images for Ge epitaxy on compressively strained 2 ML Ge and on a relaxed 3D Ge island, respectively. The image size and the growth temperature are the same. The difference in the island density (2D islands) is clearly visible. The difference in the visible size of magic clusters is related to the tip condition. On both surfaces a $(7 \times 7)$ surface reconstruction is present. For a quantitative analysis of the effect of strain on diffusion, several series of deposition were performed for a range of temperatures.

Figure 7 shows the temperature dependence of the 2D island density of $\mathrm{Ge}$ islands grown on a compressively strained and a relaxed Ge surface. In Ge epitaxy on the
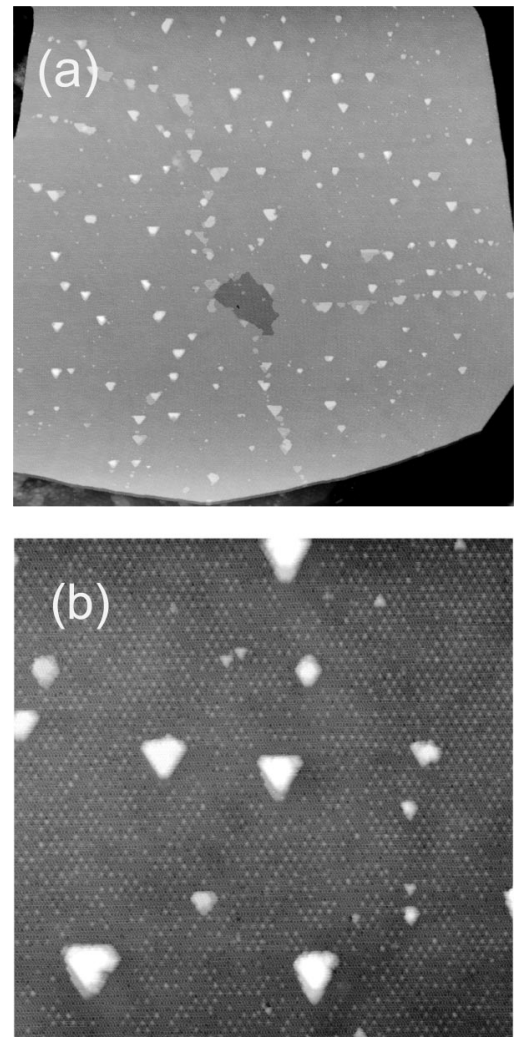

FIG. 5. STM images of 0.3 ML Ge grown at $485 \mathrm{~K}$ on a 3D Ge island on $\mathrm{Si}(111)$. (a), $7100 \times 7100 \AA^{2}$. (b), $1770 \times 1770 \AA^{2}$. "Magic" clusters and usual $2 \mathrm{D}$ islands are present on the $(7 \times 7)$-reconstructed 3D island top.

strained surface the island density increases $4-5$ times relative to the relaxed surface.

Si 2D islands grown on strained and relaxed Ge surfaces have a reversed inner strain compared to the case of $\mathrm{Ge}$ islands. Si islands on relaxed Ge surface are under tensile stress whereas Ge islands are relaxed. The density of Si islands on strained $\mathrm{Ge}$ and relaxed Ge surfaces shows the same tendency as for the case of Ge islands, see Fig. 8. Si 2D islands on a (tensile) strained surface also have increased density compared to a relaxed surface. The difference in island density is about two times in this case.

According to the Venables theory of nucleation, there are several important parameters which define the density of islands at given temperature and growth rate- the activation energy of surface diffusion $\left(E_{d}\right)$, the binding energy between a pair of atoms $\left(E_{b}\right)$, and the energy of the critical cluster of size $i\left(E_{i}\right)$.

In the following we give qualitative arguments how the binding energy of a Ge nucleus changes with the strain of the substrate. Ge islands on a strained Ge surface are under strong compressive stress. Therefore, it is easier for atoms to detach from such an island than from a nonstrained island. This means that the lateral $E_{b}$ (and finally $E_{i}$ ) for atoms in such an island is lower compared to an island on a relaxed surface. The lowering of $E_{i}$ leads to a decrease of the resulting island density on a strained Ge surface. Our experiment shows the opposite behavior, on the compressively strained surface the island density is increased. Therefore, the change 

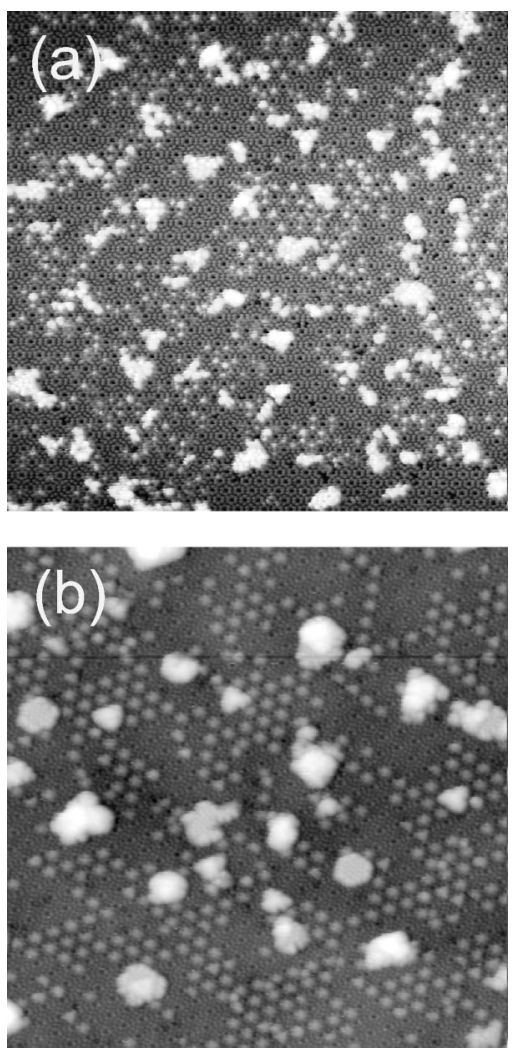

FIG. 6. STM images $\left(880 \times 880 \AA^{2}\right)$ of Ge islands grown at 430 $\mathrm{K}$ (a) on compressively strained $2 \mathrm{ML} \mathrm{Ge}$, and (b) on a relaxed 3D Ge island.

of the binding energy with strain has to be a minor effect. The observed increase of the island density on the strained substrate can be explained by an increased diffusion barrier $\left(E_{d}\right)$ on the strained surface.

The sign of the change of $E_{b}$ is different for the case of $\mathrm{Si}$ epitaxy on strained Ge, and the same reasoning is not appropriate. But due to similar nature of $\mathrm{Si}$ and $\mathrm{Ge}$ atoms and the same tendency of island density changing, we expect the same behavior of $E_{d}$ for the Si case also.

To obtain a numerical estimate of $E_{d}$, it is necessary to know the critical nucleus size and its energy, which are un-

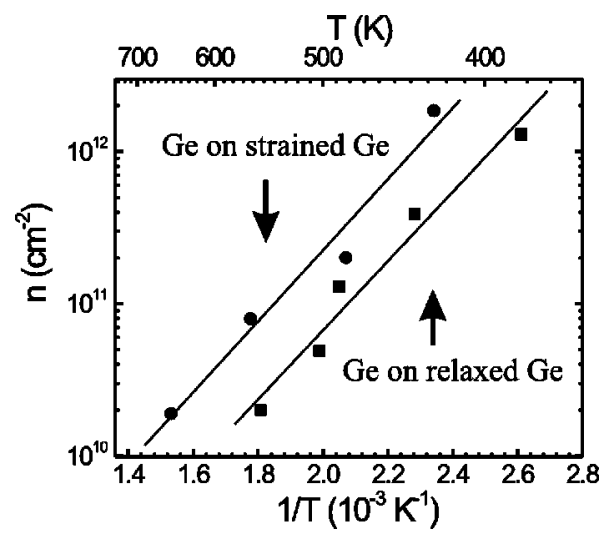

FIG. 7. Island density of $2 \mathrm{D} \mathrm{Ge}$ islands grown on a compressively strained and relaxed Ge surface.

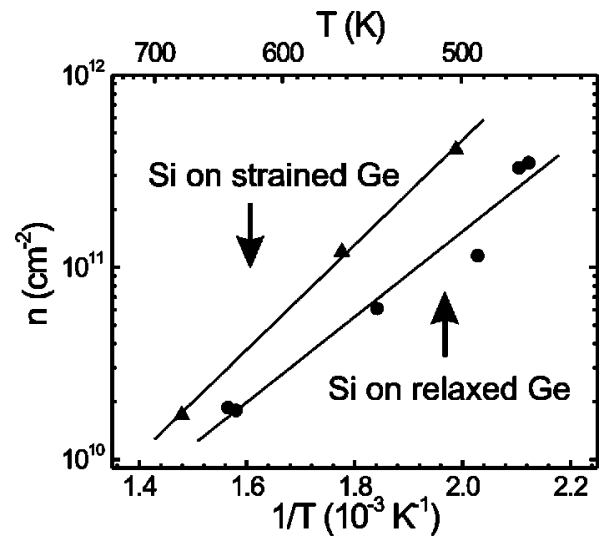

FIG. 8. Island density of $2 \mathrm{D} \mathrm{Si}$ islands grown on a compressively strained and a relaxed Ge surface.

known. However, under some assumptions the relative change of the diffusion energy between strained and relaxed surfaces can be estimated. First, we assume that the size of the critical nucleus is (nearly) the same for these two surfaces. There are experimental measurements which confirm this assumption. ${ }^{20,21}$ The second assumption is that the change of $E_{i}$ with strain is negligible, because it results in an effect which is opposite to the observed one. For a first-order estimate (lower limit) we assume that $E_{i}$ is equal on strained and unstrained surfaces. Finally the preexponential factors in the island density dependence differ not significantly (not more than 2-3 times). In frame of above assumptions the relative change of the activation energy for surface diffusion can be calculated from Eq. (1) as the difference of the slopes in the Arrhenius plot multiplied by the coefficient $i /(i+2)$. Quantitatively it is estimated that the barrier for Ge atoms on a $\mathrm{Ge}(111)-(7 \times 7)$-reconstructed surface is $50 \mathrm{meV}$ (for $i$ $\gg 1$ ) and $70 \mathrm{meV}$ (for $i=5$ ) higher on the compressively strained surface than on the relaxed surface. Corresponding calculations for the case of Si epitaxy on $\mathrm{Ge}(111)-(7 \times 7)$ surface resulted in a similar increased value of the diffusion energy on a strained surface.

We also tried to prepare a strained Si surface to study the effect of tensile strain on surface diffusion. Several experiments were performed with deposition of $\mathrm{Si}$ on $3 \mathrm{D}$ Ge islands with variety of growth temperatures and film thicknesses. The aim was to grow a smooth tensile strained Si film on the 3D Ge island. But due to the significant tensile stress the Si film formed a surface reconstruction different from either the $(7 \times 7)$ or the $(5 \times 5)$.

Figure 9(a) shows the typical surface morphology of a $\mathrm{Si}$ film on top of a 3D Ge island. The Si film has a random net of trenches formed by rows of missing atoms; in between these trenches domains of various reconstructions are observed. The trenches allow to relax the film partly. The missing rows of atoms seem to form a barrier for the diffusing adatoms. In experiments with consecutive deposition of a submonolayer coverage of $\mathrm{Si}$ the images show that location of the nucleated islands strongly correlates with the location of the trenches, Fig. 9(b). The islands nucleate preferentially at the trenches of missing atoms. One approach to prevent the formation of trenches is to grow $\mathrm{Ge}_{x} \mathrm{Si}_{1-x}$ alloys to pro- 

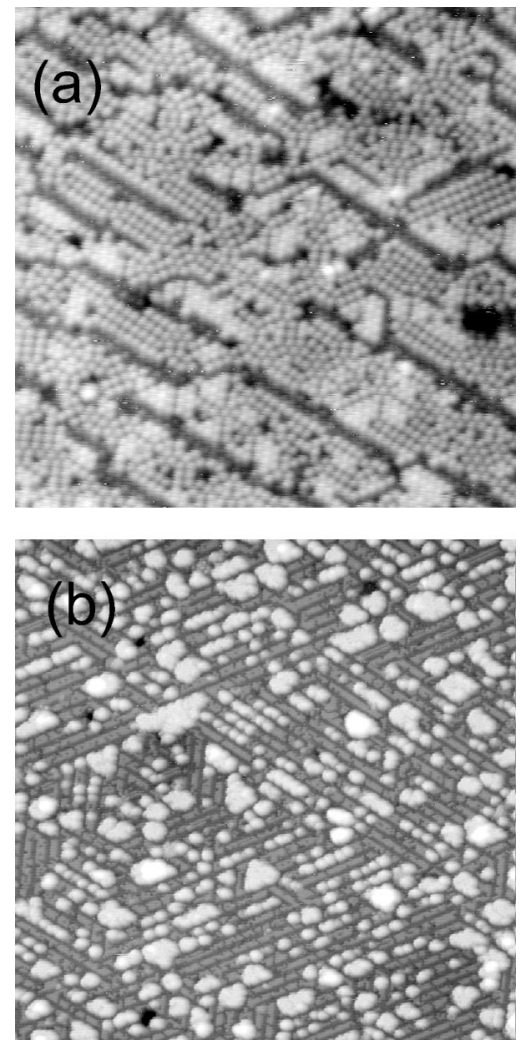

FIG. 9. STM images of (a) $\left(443 \times 443 \AA^{2}\right) 4$ ML thick Si film grown on $3 \mathrm{D} \mathrm{Ge}$ island at $810 \mathrm{~K}$. (b) $\left(1330 \times 1330 \AA^{2}\right) \mathrm{Si}$ islands grown at $620 \mathrm{~K}$ on surface shown in (a).

duce 3D islands, which have less lattice mismatch with $\mathrm{Si}$. A Silicon thin film grown on top of such 3D islands will be less strained compared to $\mathrm{Si}$ on the top of a pure Ge 3D island and may have a $(7 \times 7)$ reconstruction.

\section{INFLUENCE OF THE MATERIAL ON THE DIFFUSION LENGTH}

The diffusion barrier or the probability for an atom on a surface to jump to the next site is defined by the interaction between diffusing species and the surface. The diffusion is a complicated process, because it consists of a cooperative process of several atoms. The potential-energy surface for a surface diffusion process depends on the atom we use as a diffusing probe species and on the substrate material. We performed island density measurements to clarify the relation between diffusion and the material $(\mathrm{Si}$ or $\mathrm{Ge})$ used as the diffusing species and $\mathrm{Si}$ or Ge used as substrate material. Figure 10(a) presents the $\mathrm{Si}$ and $\mathrm{Ge}$ island densities in an Arrhenius plot after submonolayer growth on $(7 \times 7)-\operatorname{Si}(111)$ and on top of relaxed 3D Ge islands. The strong material dependence of the island densities is clearly visible. In Fig. 10(b) the different material combinations are sketched schematically. Si islands on a Si substrate (1) have the highest density, whereas Ge islands on Ge (4) reveal the lowest island density. The difference between those two cases is about two orders of magnitude.

If we keep the substrate material the same and change the
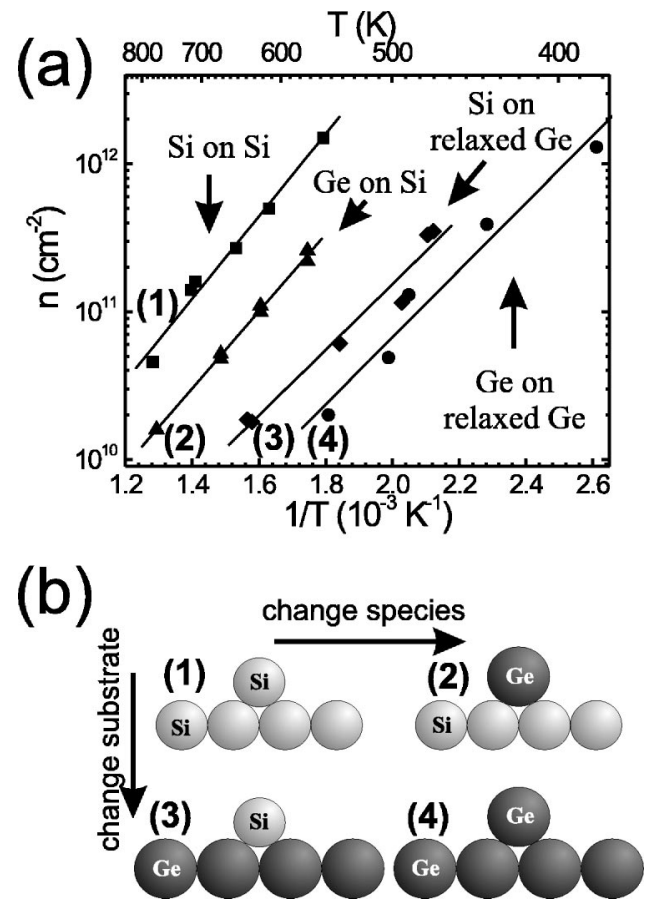

FIG. 10. (a) Island density as a function of temperature for epitaxy combination $\mathrm{Si}$ and $\mathrm{Ge}$ as a deposited material and substrate. (b) Schematic of cases (1)-(4) in (a).

deposited element from $\mathrm{Si}$ to Ge by going from material combination $(1) \rightarrow(2)$, or $(3) \rightarrow(4)$, in Fig. 10, in both cases the island densities decrease, which shows an increase of the diffusion length of Ge atoms on those surfaces compared to diffusing Si atoms. The island densities increase by a factor of about $2-3$ by changing the diffusing species from Si to Ge.

If we change the substrate material and keep the deposited element the same by going from material combination (1) $\rightarrow(3)$, and (2) $\rightarrow$ (4) in Fig. 10, the island density decreases about one order of the magnitude in both cases. This shows that the influence of the substrate material on the diffusion length is much stronger than the influence of the diffusing species. Again the diffusion length increases when $\mathrm{Si}$ is replaced by Ge (as substrate material in this case).

Summarizing the results, the diffusion length increases when $\mathrm{Si}$ is replaced by Ge as deposited material or as substrate material. The influence of the substrate material on the diffusion length is much stronger (factor 10 in the island density) than the influence of the adsorbed material (factor $2-3)$. In the following we will discuss these results. We will consider several possible mechanisms to explain the observed island densities. We will consider the influence of only one single mechanism on the diffusion length separately, while we neglect the influence of the other mechanisms (for the moment). If the considered mechanism can not account qualitatively for the observed behavior, the influence of this mechanism will considered to be small.

As a first mechanism we consider the strain induced change of the diffusion barrier. From comparison of $\mathrm{Si}$ on $\mathrm{Si}$ and $\mathrm{Ge}$ on $\mathrm{Ge}$, which are both unstrained systems, we see immediately that the influence of the material (factor 100 in 
island density) is much larger than the influence of strain (c.f. Fig. 7).

A second mechanism potentially influencing the diffusion length is the strain contribution to the binding energy of the critical nucleus. As outlined in the preceding section this effect should lead to a decrease of the island density when the deposited material is under stress. A larger diffusion length is expected on a strained surface (compressive or tensile strained) and a smaller diffusion length is expected on the relaxed surface. If we neglect the different chemical nature of $\mathrm{Si}$ nd $\mathrm{Ge}$ (for the moment) and consider only the strain we can see that in experiment the behavior is opposite, a smaller diffusion length (larger island density) on the tensile strained $\mathrm{Si}$ on $\mathrm{Ge}$ [case (3) in Fig. 10] compared to the relaxed case $\mathrm{Ge}$ on Ge [case (4) in Fig. 10]. Therefore, also this mechanism can be excluded as the dominating one.

Another mechanism is the bonding energy of the critical nucleus due to the different bonding strength of $\mathrm{Ge}$ and $\mathrm{Si}$. Measurements of the rate dependence of the 2D island density showed that the critical nucleus size is similar in $\mathrm{Si}$ and Ge epitaxy. ${ }^{20,21}$ Critical nucleus size $i$ for $\mathrm{Si}$ on $\mathrm{Si}$ is 7, and for $\mathrm{Ge}$ on $\mathrm{Si} i$ is 8. Therefore, mainly the different bonding strengths of $\mathrm{Si}$ and Ge determine the bonding energy of the critical cluster. It is known, for instance, from comparison of the cohesive energies of $\mathrm{Ge}$ and $\mathrm{Si}$ that the bonding of $\mathrm{Si}$ is stronger than that of Ge. Therefore, the binding energy of the critical nucleus of Si will be larger than that of Ge. This leads to a smaller diffusion length of Si compared to Ge as diffusing species. This is in accord with the observed behavior going from case (1) to case (2) and from case (3) to case (4), which corresponds to an increase of the diffusion length upon the change from $\mathrm{Si}$ as diffusing species to Ge. Therefore, the different bonding strengths of $\mathrm{Si}$ and $\mathrm{Ge}$ are a mechanism which is consistent with the observed behavior during the change of the diffusing species.

Another possible mechanism which can influence the dif- fusion length is the influence of the element (substrate or diffusing species) on the diffusion barrier of the diffusing species. Generally it is known that the bonding strength is related to the diffusion barriers at surfaces. The stronger the bonding energy of an element is, the larger will be the diffusion barrier. This general rule is in accord with the observed trend of longer diffusion length whenever $\mathrm{Si}$ is replaced by $\mathrm{Ge}$ (as diffusing species or as substrate material), $(1) \rightarrow(2),(3) \rightarrow(4),(1) \rightarrow(3)$, and $(3) \rightarrow(4)$. The enhanced effect upon the change of the elements in the substrate can be explained by the replacement of more atoms from Si to Ge than for the case of the change of the diffusing species, which corresponds only to a change of one atom taking part in the diffusion event from $\mathrm{Si}$ to Ge.

Summarizing this part, the influence of the material on the diffusion length is dominated by the different bonding strengths of $\mathrm{Si}$ and $\mathrm{Ge}$ in the island and with the substrate. The influence solely due to strain is much smaller.

\section{CONCLUSION}

Dedicated templates which are different only in one particular feature, reconstruction, or strain, or material, are prepared to study the dependence of the surface diffusion length on these properties. Island density measurements as a function of temperature on strained and relaxed Ge(111) surfaces have been made to study the effect of strain on the surface diffusion. The barrier for diffusion was found to increase on the compressively strained surfaces. Island densities on (5 $\times 5)$ - and $(7 \times 7)$-reconstructed surfaces are almost equal in spite of presence significant number of magic clusters on $(7 \times 7)$-reconstructed surface. The change of the substrate from $\mathrm{Ge}$ to $\mathrm{Si}$ changes the island density greatly. An increase of the diffusion length is observed when $\mathrm{Si}$ is replaced by Ge as substrate material, or as diffusing species. This trend is explained by the stronger bonding of Si compared to Ge.
${ }^{1}$ A.I. Nikiforov, V.A. Markov, V.A. Cherepanov, and O.P. Pchelyakov, Thin Solid Films 336, 183 (1998).

${ }^{2}$ Y.-W. Mo and M. Lagally, Surf. Sci. 248, 313 (1991).

${ }^{3}$ Y.-W. Mo, J. Kleiner,. M.B. Webb, and M.G. Lagally, Surf. Sci. 268, 275 (1992).

${ }^{4}$ B.S. Swartzentruber, Phys. Rev. Lett. 76, 459 (1996).

${ }^{5}$ H.J.W. Zandvliet, B. Poelsema, and B.S. Swartzentruber, Phys. Today 54 (7), 40 (2001).

${ }^{6}$ H. Brune, K. Bromman, H. Röder, J. Jacobsen, P. Stoltze, K. Jacobsen, and J. Norskov, Phys. Rev. B 52, R14 380 (1995).

${ }^{7}$ C. Ratsch, A.P. Seitsonen, and M. Scheffer, Phys. Rev. B 55, 6750 (1997).

${ }^{8}$ M. Schroeder and D.E. Wolf, Surf. Sci. 375, 129 (1997).

${ }^{9}$ T. Hoshino, M. Hata, and M. Tsuda, Surf. Sci. 481, 205 (2001).

${ }^{10}$ J.A. Venables, Surf. Sci. 299/300, 798 (1994).

${ }^{11}$ D.-S. Lin, H. Hong, T. Miller, and T.-C. Chiang, Surf. Sci. 312, 213 (1994).

${ }^{12}$ N. Motta, A. Sgarlata, R. Calarco, Q. Nguyen, J. Castro Cal, F.
Patella, A. Balzarotti, and M. De Crescenzi, Surf. Sci. 406, 254 (1998).

${ }^{13}$ P.W. Deelman, L.J. Schowalter, and T. Thundat, J. Vac. Sci. Technol. A 15, 930 (1997).

${ }^{14}$ N. Paul, H. Asaoka, J. Mysliveckek, and B. Voigtlander (unpublished).

${ }^{15}$ H.-J. Gossmann, J.C. Bean, L.C. Feldman, E.G. McRae, and I.K. Robinson, Phys. Rev. Lett. 55, 1106 (1985).

${ }^{16}$ R.M. Tromp, Phys. Rev. B 47, 7125 (1993).

${ }^{17}$ I.-S. Hwang, M.-S. Ho, and T.-T. Tsong, J. Phys. Chem. Solids 62, 1655 (2001).

${ }^{18}$ H. Asaoka and B. Voigtländer (unpublished).

${ }^{19}$ C.M. Chang and C.M. Wei, Phys. Rev. B 67, 033309 (2003).

${ }^{20}$ L. Andersohn, Th. Berke, U. Köhler, and B. Voigtländer, J. Vac. Sci. Technol. A 14, 312 (1996).

${ }^{21}$ S.A. Teys and B.Z. Olshanetsky, in Proceedings of the 12th International Conference on STM/STS and Related Techniques (unpublished). 